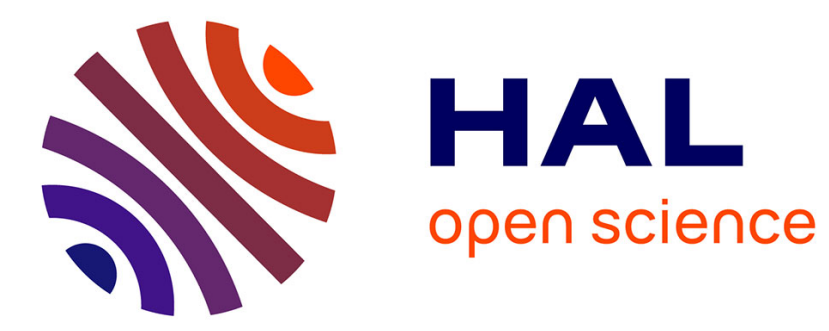

\title{
Les victimes écrivent leur histoire
}

Sandrine Lefranc, Lilian Mathieu, Johanna Siméant

\section{To cite this version:}

Sandrine Lefranc, Lilian Mathieu, Johanna Siméant. Les victimes écrivent leur histoire. Raisons politiques, 2008, 30, pp.5-20. 10.3917/rai.030.0005 . halshs-00828330

\section{HAL Id: halshs-00828330 https://shs.hal.science/halshs-00828330}

Submitted on 12 Jan 2018

HAL is a multi-disciplinary open access archive for the deposit and dissemination of scientific research documents, whether they are published or not. The documents may come from teaching and research institutions in France or abroad, or from public or private research centers.
L'archive ouverte pluridisciplinaire HAL, est destinée au dépôt et à la diffusion de documents scientifiques de niveau recherche, publiés ou non, émanant des établissements d'enseignement et de recherche français ou étrangers, des laboratoires publics ou privés. 


\title{
Les victimes écrivent leur Histoire Introduction
}

\author{
SANDRINE LEFRANC, LILIANMATHIEU \\ ET JOHANNA SIMÉANT
}

\section{$\mathrm{L}$} A FIGURE DE LA VICTIME a connu en quelque trois décennies une consécration sociale incontestable, comme le soulignent Didier Fassin et Richard Rechtman en parlant d'une « innovation sociale majeure » et d'une « reconfiguration de l'économie morale contemporaine » ${ }^{1}$. Ce dossier, qui réunit cinq exemples de mobilisations de « victimes », voudrait revenir sur cette reconnaissance sociale des victimes devenue évidence, en montrant notamment en quoi la constitution de causes de victimes, pas plus que toute autre forme de mobilisation sociale, ne va de soi, et est un problème scientifique difficile à résoudre pour ceux qui s'en emparent. Comment rendre compte scientifiquement de processus qui se donnent à voir socialement à travers le filtre de qualifications morales : " victime », bien sûr, mais aussi, ici, « violence » ? Que faire de l'hypothèse d'une spécificité de mobilisations «victimaires » s'il s'agit d'une simple qualification (qui échoue ou «prend » sur le plan social) et que toute souffrance la " mérite »?

Le qualificatif de victime peut désormais avoir la valeur d'un statut : par exemple, titre à faire valoir pour l'obtention de prestations

1. « La découverte de cette mémoire douloureuse est un fait anthropologique majeur des sociétés contemporaines ", Didier Fassin et Richard Rechtman, L'Empire du traumatisme. Enquête sur la condition de victime, Paris, Flammarion, p. 29. 
administratives, ou rôle à jouer sur une scène judiciaire qui il y a peu conférait à la victime du crime une place tout à fait mineure. L'une des formes prises par cette reconnaissance, qui est aussi l'une de ses origines, concerne le rapport d'une société donnée à un passé de violence politique. Un « droit» de faire entendre son récit propre, et de participer à l'écriture de l'Histoire, semble avoir été octroyé à « la victime ». C'est ce qui peut être déduit de la multiplication des récits écrits sur la base de témoignages ou de thérapies, et l'intégration croissante de ces récits dans des dispositifs professionnels nombreux ; Fassin et Rechtman le montrent en matière de gestion des attentats et catastrophes naturelles ainsi que des demandes d'asile en France, mais aussi dans le secteur de l'intervention humanitaire d'urgence dans les pays étrangers. Ils en tirent une conclusion de portée générale : «Au cours du dernier quart de siècle, le traumatisme s'est imposé comme une forme d'appropriation originale des traces de l'histoire et comme un mode de représentation dominant du rapport au passé ${ }^{2}$. » Ce constat a connu des formulations antérieures, celle par exemple d'un " devoir de mémoire » pensé comme réparation à l'égard des victimes.

Cette prise en compte croissante de la parole « subjective » des victimes concerne donc aussi bien les formes d'intervention sociale qui se donnent pour non gouvernementales que des dispositifs gouvernementaux. Dans des contextes d'après-guerre et de fin de période de répression, l'accord de nombreux gouvernements dits de transition, d'organisations non gouvernementales et d'organisations internationales autour du dispositif de commission de vérité et de réconciliation, en est un bon exemple, dans la mesure où cette convergence est justifiée le plus souvent par la capacité de ces commissions - mieux que les réunions d'historiens ou les commissions parlementaires - à intégrer dans leur rapport la " vérité subjective » des victimes ${ }^{3}$. D’autres techniques articulant cette vérité et les histoires scientifiques ou " officielles » connaissent un développement important, comme les manuels d'Histoire écrits à quatre mains, censées représenter les ennemis d'hier ${ }^{4}$.

Ces formes relativement nouvelles d'articulation de différents

2. Ibid.

3. Voir Sandrine Lefranc (dir.), Après le conflit, la réconciliation ?, Paris, Michel Houdiard, 2006, et, du même auteur, « La consécration internationale d'un pis-aller : une genèse des politiques de "réconciliation" ", in Georges Mink et Laure Neumayer, L'Europe et ses passés douloureux, Paris, La Découverte, 2007, p. 233-247.

4. Voir par exemple, à l'initiative du Peace Research Institute in the Middle East 
récits sur le passé (de l'Histoire et de la mémoire, diraient d'autres), ne garantissent pas pour autant leur concordance. Quand les victimes écrivent leur Histoire, les sciences sociales applaudissent à la fin de la domination du récit du bourreau, mais veillent aussi à défendre leur monopole sur l'écriture d'une Histoire objective et commune. Cette tension se résout souvent dans l'adoption de la figure d'une «bonne victime » qui, acceptant que l'Histoire soit objectivée, ou du moins qu'elle fasse une place aux récits des autres (autres victimes, voire bourreaux), demeure raisonnable dans ses revendications comme dans l'usage d'un registre émotionnel. La recommandation de l'historien David Anderson ${ }^{5}$, lorsqu'il propose que tous les squelettes de victimes de l'époque coloniale au Kenya soient, quelle qu'ait été la position de ces dernières, enterrés ensemble dans un Heroes Acre, est emblématique de cette raison des sciences sociales que toutes les victimes de violence politique ne font pas leur ${ }^{6}$.

Cette raison du scientifique est l'héritière d'une longue histoire, celle de la construction d'une figure de l'universitaire honnête homme qui réprouve, moralement, la description de la souffrance, et prend scientifiquement ses distances d'avec le monde social ; Luc Boltanski a montré comment les sciences sociales se sont donné pour principe de remettre en question le caractère spontané et gratuit des émotions pour dévoiler les stratégies rationnelles qu'elles recouvrent ${ }^{7}$. Mais la fascination pour la souffrance et l'impératif d'une position libérale d'empathie sont aussi le produit d'une histoire plus courte. C'est depuis le génocide des Juifs perpétré lors de la Deuxième Guerre mondiale, en effet, que les historiens ont, après avoir longtemps omis la principale victime de la violence nazie, consacré la "victime ", point de mire du tableau historique et, en

(PRIME), Histoire de l'autre, Paris, Liana Levi, 2004, trad. de l'hébreu par Rosie Pinhas-Delpuech et de l'arabe par Rachid Akelet. L'ouvrage est issu du travail conjoint de six enseignants d'histoire palestiniens, six autres israéliens, six «délégués internationaux » et un observateur israélien.

5. Évoquée par Marie-Émmanuelle Pommerolle dans ce numéro.

6. Le refus, pleinement politique, de toute forme de commémoration et d'hommage par les Mères argentines de la place de Mai (ligne Hebe de Bonafini) est caractéristique de cette résistance.

7. Luc Boltanski, La Souffrance à distance. Morale humanitaire, médias et politique, Paris, Métailié 1993, p. 127-128. L'ouvrage dirigé par Christophe Traïni sur la place des émotions (dans le cadre de "dispositifs de sensibilisation ») dans les mouvements sociaux, à paraître aux Presses de Sciences Po en 2008, donne la mesure de l'intérêt de la prise en compte de ces émotions aussi bien que de sa difficulté. 
tant que témoin, source de connaissance. L'événement a donc contribué à une reformulation des règles du jeu de l'écriture historique, au profit notamment de la singularisation de ce récit. Celle-ci prend place d'ailleurs dans un " air du temps » plus général, qui, par exemple dans le cadre du mouvement en faveur de formes de délibération politique, semble donner une grande importance aux récits les plus subjectifs ${ }^{8}$. Mais ce bouleversement connaît des limites, puisque ce sont les mêmes historiens qui condamnent souvent vigoureusement les « concurrences victimaires » et autres affirmations jugées instrumentales et particularistes du point de vue de la victime ; Hilberg, Nora, Todorov, Ricœur, Chaumont et d'autres convergent sur ce point en pointant les « abus » qui surgiraient trop souvent, et sitôt que la mémoire se ferait politique ${ }^{9}$. Ils soulignent la nécessité du passage d'une mémoire privée à une mémoire publique - convertissant le «bruit» de la plainte en un langage éthico-juridique susceptible de rompre le cycle du ressentiment et l'enfermement dans une identité close.

Les historiens, et plus largement les sciences sociales, ne se déprennent donc pas aussi aisément qu'on pourrait le croire d'un contexte social qui, consolidant ou non un groupe porteur de mémoire, participe de la consécration d'une figure de la victime, mais agit encore " comme la matrone d'Éphèse, qui pend le mort pour sauver le vivant ${ }^{10} »$. Maurice Halbwachs montrait par cette métaphore comment une mémoire collective ne pouvait survivre à

8. On fait référence ici aux critiques féministes du modèle délibératif qui, avec Alex Honneth, mettent notamment l'accent sur les exigences de l'expression de la souffrance. Voir Nancy Fraser, Qu'est-ce que la justice sociale ?, trad. et introd. par Estelle Ferrarese, Paris, La Découverte, 2005 (1992) ; Iris Marion Young, Inclusion and Democracy, Oxford, Oxford University Press, 2000 ; Axel Honneth, La Société du mépris. Vers une nouvelle théorie critique, trad. de l'all. par Olivier Voirol, Pierre Rusch et Alexandre Dupeyrix, Paris, La Découverte, 2006.

9. Voir Raoul Hilberg, La Politique de la mémoire, trad. de l'angl. par Marie-France de Paloméra, Paris, Gallimard, 1996, p. 127 et suiv., critiquant la « campagne de glorification " dont feraient l'objet toutes les victimes de la Shoah. Voir aussi Tzvetan Todorov, Les Abus de la mémoire, Paris, Arléa, 1995, notamment p. 57 et suiv., et Jean-Michel Chaumont, La Concurrence des victimes, Génocide, identité et reconnaissance, Paris, La Découverte, 1991, notamment p. 320 et suiv. Selon T. Todorov, «le culte de la mémoire ne sert pas toujours de bonnes causes (...) il peut être l'expression du conservatisme et de la survalorisation de l'identité », peut alimenter le populisme ou le misérabilisme, le nationalisme ou l'esprit de vengeance, «La mémoire et ses abus », Esprit, n 193, juillet 1993, p. 34-44. Il y a « un mérite incontestable de passer de son propre malheur, ou de celui de ses proches, au malheur des autres, de ne pas réclamer pour soi le statut exclusif [...] de l'ancienne victime », ibid., p. 42.

10. Maurice Halbwachs, Les Cadres sociaux de la mémoire (1925), Paris, Albin Michel, 
l'évanouissement du groupe qui naguère la portait. Et ce qui vaut pour l'individu privé de soutien, vaut pour la " première » victime, celle qui, entrant (toujours avec d'autres, bien sûr) dans la mobilisation, ne peut se faire entendre que si elle s'appuie sur un groupe social, constitué dans la mobilisation. Au-delà de l'appréciation morale d'un «droit » des victimes à faire entendre et faire reconnaître leur récit historico-mémoriel, le problème posé ici est de comprendre comment " prend », sur un plan collectif, une prétention à faire entendre une " histoire non-écrite " ${ }^{11}$ et à se faire qualifier de victime.

\section{La constitution des groupes}

Parler de "mobilisation de victimes » expose en effet à un péril : celui de présupposer réglé le problème du groupe-emblème de la protestation et de sa délimitation, de tenir pour acquis le processus de rassemblement d'individus dans un collectif en quoi réside précisément la mobilisation. C'est, en d'autres termes, supposer résolu, et négliger de traiter, ce qui constitue pour les entrepreneurs de cause un problème majeur, celui de la construction et de la consolidation du groupe dont ils se prétendent les défenseurs ou les représentants. Si cette question des modes de production du collectif se pose pour toute étude de mobilisation, elle n'en est pas moins susceptible de revêtir, dans le cas des victimes de violences politiques, un certain nombre de traits saillants.

Le double travail « de regroupement, d'inclusion et d'exclusion » et de "définition et de délimitation » au principe de la formation des groupes, qu'évoque Luc Boltanski ${ }^{12}$, peut ainsi s’avérer

1994, p. 167. L'expression est précédé par ce passage : «L’individu qui ne veut pas oublier ses parents disparus, et s'obstine à répéter leurs noms, se heurte assez vite à l'indifférence générale. Muré dans ses souvenirs, il s'efforce en vain de mêler aux préoccupations de la société actuelle celles des groupes d'hier : mais il lui manque précisément l'appui de ces groupes évanouis. Un homme qui se souvient seul de ce dont les autres ne se souviennent pas ressemble à quelqu'un qui voit ce que les autres ne voient pas. C'est, à certains égards, un halluciné, qui impressionne désagréablement ceux qui l'entourent. Comme la société s'irrite, il se tait, et à force de se taire, il oublie les noms qu'autour de lui personne ne prononce plus ».

11. L'expression renvoie à Antigone qui, de Sophocle à Anouilh, invoque des lois «non écrites ".

12. Luc Boltanski, Les cadres. La formation d'un groupe social, Paris, Minuit, 1982, p. $51-52$. 
particulièrement ardu lorsque font défaut les instruments nécessaires à la production du collectif, ou lorsque l'état du rapport de force qui oppose à un oppresseur reste défavorable, et tout spécialement lorsque celui-ci tend à cantonner la victime à une expérience singulière de son malheur. L'intérêt, de ce point de vue, de l'article d’Henry Sorg est précisément de porter sur une situation que l'on pourrait qualifier de proto- ou d'infra-mobilisation. L'expérience de la perte d'un proche dans les massacres de prisonniers politiques de 1988 reste avant tout une expérience singulière, vécue dans une intimité douloureuse, et dont le régime iranien vise précisément à empêcher le partage. Par l'entretien d'une incertitude sur les lieux d'inhumation, l'exclusion symbolique d'une communauté d'identification (celle de l'islam), l'intimidation et l'isolement des proches, le régime a entrepris d'entraver la connexion et l'association entre proches des victimes, mais sans cependant pouvoir totalement freiner un processus d'ébauche de collectif centré sur des cérémonies de commémoration en des sites-symboles. Contexte défavorable à la consolidation du groupe, également, dans le cas des épurés de l'après-guerre : comme tout stigmate, celui de " collabo » exige un contrôle attentif de l'information sur ses traits potentiellement disqualifiants, qui ne peut être exposée que dans des médias au statut de « lieux retirés " ${ }^{13}$, et encore sous la forme euphémisée et semiclandestine d'abréviations lisibles des seuls initiés. Et lorsque le régime se fait moins oppressif, c'est bien souvent sous une forme individualisée - comme les réhabilitations individuelles consenties aux Tatars par le régime soviétique - qu'il livre ses concessions, faisant de la sorte obstacle à toute reconnaissance d'un dommage subi collectivement.

Autrement dit, une communauté d'expérience d'une injustice ou d'un malheur entre individus ne préjuge en rien de leur capacité à exprimer collectivement une exigence de reconnaissance et de réparation. Ainsi que le souligne dans son article Eric Savarese, des personnes ayant subi le même dommage forment un groupe circonstanciel ${ }^{14}$ bien avant que de constituer un groupe de lutte, et il

13. Les lieux retirés sont pour Goffman des sites « où le stigmate est accepté librement, sans voiles »; Stigmate, Les usages sociaux des handicaps (1963), trad. de l'angl. par Alain Kihm, Paris, Minuit, coll. "Le Sens Commun », 1975 p. 100.

14. Bien que ces auteurs ne traitent pas spécifiquement des victimes de violence politique, le terme est emprunté à Jean-Paul Vilain et Cyril Lemieux, "La mobilisation des victimes d'accidents collectifs. Vers la notion de "groupe circonstanciel” ", Politix, vol. 11, no 44, 1998, p. 135-160. 
est fréquent que, même lorsqu'une mobilisation se développe en leur nom collectif, un nombre important d'entre elles en resteront à l'écart (pour des raisons pouvant aller du sentiment d'incompétence politique à l'anticipation des divers coûts de l'engagement). De même n'est-il pas assuré que la base sur laquelle s'opère le ralliement à la cause, lorsque celle-ci parvient à émerger, soit toujours de nature politique - ainsi que l'indiquent les services d'entraide, fonctionnant comme autant d'incitations sélectives, que proposent aux épurés les journaux d'extrême droite de l'après-guerre (Caroline Baudinière).

Une " communauté de destin », en d'autres termes, ne suffit pas à elle seule à impulser la constitution et la consolidation d'un groupe, encore faut-il un travail spécifique dont il convient d'étudier les modalités et les acteurs. La distinction qu'opérait Bourdieu entre " classe probable », définie par le partage d'un certain nombre de traits, et «classe mobilisée », dotée d'une assise collective et de représentants habilités à parler en son nom ${ }^{15}$, s'avère ici utile, en pointant que c'est précisément via la délégation du pouvoir de représentation à des porte-parole qu'une population antérieurement dispersée et dépourvue d'identité propre peut accéder à l'existence politique.

Plusieurs des textes réunis dans ce dossier témoignent des limites et des ambivalences qu'implique toute logique de délégation. Ces limites sont évidentes lorsque la principale victime est elle-même absente et ne peut témoigner de la nature des supplices qu'elle est supposée avoir subis. La disparition, l'absence des corps, laissent planer une incertitude sur la réalité de l'injustice dénoncée, dans le même temps qu'elles impliquent un déplacement du statut victimaire, de la victime à ses proches qui, eux, souffrent de son absence et de l'incertitude sur son sort. Se pose ainsi de manière centrale la question de la légitimité des porte-parole à s'exprimer au nom d'un groupe totalement ou partiellement composé d'absents, elle-même indissociable de la question de sa représentativité. La sélection des porte-parole selon leur degré d'aisance à s'exprimer publiquement et à prendre en charge des tâches organisationnelles, ici comme ailleurs, reproduit et consolide les inégalités internes au groupe, dans le même temps qu'elle induit un processus de professionnalisation, ou tout au moins de spécialisation, favorisant l'autonomisation des porte-parole

15. Pierre Bourdieu, «Espace social et genèse des "classes” ", Actes de la recherche en sciences sociales, $\mathrm{n}^{\circ}$ 52-53, 1984, p. 3-12. 
à l'égard de ceux qu'ils représentent. L'article de Marie-Émmanuelle Pommerolle donne dans le dossier une illustration exemplaire des risques de dépossession, toujours à l'état potentiel dans la délégation, en montrant combien la cause des vétérans Mau Mau a été appropriée par une diversité d'acteurs se réclamant de l'héritage (et de la légitimité) de leur lutte pour des enjeux spécifiques à la compétition politique kenyane contemporaine. Loin de se mobiliser elles-mêmes, les victimes sont ici mobilisées avec le statut de ressource symbolique dans des combats auxquels elles restent largement étrangères.

Sans doute du fait de la gravité des violences subies, qui peut faire déboucher le doute systématique sur une posture assimilée au révisionnisme, les groupes de victimes sont trop souvent considérés comme acquis, ce qui fait trop souvent négliger leur inégale consistance. Il faut pourtant envisager les dimensions qui contribuent à les homogénéiser, et qui renvoient non seulement à des propriétés (langues, type de sociations...), mais aussi aux aspects qui ont pu contribuer à leur persécution et à leur identification. Dans les situations où la répression s'est fondée sur le repérage d'un stigmate plus ou moins facilement dévoilable, il faut ainsi travailler sur différentes hypothèses de continuité du stigmate et de sa persistance après les violences, et sur les deux faces du stigmate, comme objet d'identification étatique mais aussi comme possible élément d'appartenance au groupe. Il faut envisager également ce que les formes concrètes de la répression ont pu produire chez ses victimes, par exemple en termes de trajectoires professionnelles (dans les cas fréquents d'exclusion de l'accès à l'enseignement, au droit...), mais aussi de savoir-faire, de techniques de survie, de mobilisation et de maintien de l'identité, sur ceux qui l'ont subie : quels effets ces aspects produisent-ils sur la façon de se mobiliser ? Que sait-on de la socialisation spécifique que produisent la torture, la dénonciation, ou l'exclusion?

Que les mobilisations de victimes ne soient pas composées que de victimes invite également à se pencher sur la condition de leur pérennisation. Jusqu'à quand - combien de générations, par exemple - est-on une victime ? Les militants pieds-noirs d'aujourd'hui, fils de Français rapatriés, le sont-ils encore ? De même les enfants et petitsenfants des Tatars déportés de Crimée peuvent-ils encore se prétendre victimes de l'expulsion d'un territoire qu'ils n'ont jamais connu ? Si une approche contextualisée s'impose de toute évidence pour répondre à des questions de ce type, celle-ci ne saurait se restreindre aux seuls éléments du contexte directement liés à la 
situation victimaire initiale. Les usages contemporains par le personnel politique kenyan de la cause des Mau Mau, tout comme le débat sur la loi du 23 février 2005 portant « reconnaissance de la nation et contribution nationale en faveur des Français rapatriés ", indiquent combien les causes des victimes du passé sont indissociables des enjeux du présent, parfois largement décalés ou déconnectés, et plus encore doivent fréquemment leur survie à leur capacité à servir ces enjeux. Il importe ici de se rendre attentif aux conditions qui permettent à une cause d'être entendue, ou pas, selon les configurations historiques, médiatiques et politiques.

Parvenir à faire exister sous une forme collective les victimes au nom desquelles il s'exprime impose au représentant un double travail de définition et d'attestation du groupe. Travail de définition tout d'abord puisqu'en invoquant l'existence du groupe des victimes au principe de son statut, le porte-parole lui définit un critère de rassemblement et de cohésion, celui d'avoir subi telle injustice exigeant réparation. De sorte que faire reconnaître un groupe, et les supplices qui l'ont fait advenir, c'est tenter d'imposer sa définition de son identité contre les définitions concurrentes - et spécialement celles qui dénient la réalité de ces supplices -, c'est lui définir des contours qui, eux-mêmes, peuvent être disputés entre prétendants au statut victimaire (on sait que les homosexuels ont peiné à se faire reconnaître comme partie prenante du " groupe » des victimes du nazisme). Travail d'attestation ensuite, puisque la légitimité de tout porte-parole s'évalue à sa capacité à manifester l'existence collective du groupe au nom duquel il s'exprime. D'où le recours privilégié à des formes d'action misant sur la force du nombre et sur sa visibilité, telles que les pétitions et les rassemblements. D'où, par contrecoup, l'enjeu pour les antagonistes de la dispersion géographique (exil, déportation, enfermement...) des protestataires et de leurs proches, et, comme dans le cas iranien, l'importance des entraves à la définition de points focaux, de lieux symboliquement chargés où les proches des victimes pourraient converger et, par leur rassemblement, évaluer leur capacité collective à se mobiliser. On comprend mieux dans cette perspective l'enjeu des commémorations, le plus souvent à dates et lieux fixes, qui permettent aux membres du groupe de se rassembler et, partant, de fournir l'attestation de son existence, de se compter, de consolider sa cohésion interne et de proclamer publiquement la grandeur de son identité collective. 


\section{Des Histoires « en simili »}

Les constructions, usages et mobilisations de récits historiques par les victimes et leurs représentants, dont traitent tout particulièrement les textes qui suivent, témoignent également de ces différents enjeux. Enjeu de la production collective du groupe, tout d'abord, qui là encore impose la sollicitation de méthodes d'objectivation à même d'attester que le malheur subi ne relève pas de l'expérience singulière mais est doté d'une dimension générale ${ }^{16}$. D'où l'importance des chiffres dont les producteurs doivent construire la crédibilité de l'importance contre l'entreprise de réduction ou de dénégation des antagonistes. Le calcul du nombre de victimes de la répression coloniale au Kenya, de celui des Tatars disparus au cours de leur déportation, des opposants iraniens exécutés en 1988, ou encore des morts de l'épuration devient enjeu de débats, la crédibilité des chiffres se mesurant à la fiabilité des sources et à la rigueur des modes de calcul statistique. D'où également l'importance de ces "manœuvres pour grandir » la cause que l'on porte, comme dans le recours au terme de "génocide », en luimême porteur de la grandeur et de la légitimité de ceux officiellement reconnus comme tels.

Si l'enjeu est de toute évidence la légitimation de la cause au travers de l'invocation de son importance quantitative ou qualitative, et ainsi de sa grandeur éthique ou politique, un autre trait mérite ici l'attention : la construction isomorphe des causes antagonistes, particulièrement bien illustrée dans le cas des épurés étudié par Caroline Baudinière. Les "morts de l'épuration " sont définis comme en réponse aux fusillés du Parti communiste, de même que les images de femmes tondues sont opposées aux photos de déportés et que le centre d'interrogatoire de la Résistance est décrit par les épurés sur le modèle de celui de la Gestapo. Comme c’est généralement le cas dans les luttes de concurrence, celles entre groupes opposés induisent une construction par mimétisme, chacun entreprenant de réfuter ou de dépasser l'autre sur son propre terrain dans une logique de surenchère : Marie-Émmanuelle Pommerolle le souligne, les souffrances invoquées par les représentants des Mau Mau sont relativisées par leurs antagonistes au regard de celles qu'euxmêmes ont infligées. Mais l'isomorphisme, ou tout au moins la

16. Sur la tension entre généralité et singularité des causes, voir Luc Boltanski, L'amour et la justice comme compétences, Paris, Métailié, 1990. 
« ressemblance de famille », repérable notamment entre discours respectifs des victimes et de leurs antagonistes, peut tenir à d'autres logiques, comme l'emprise d'un univers de référence, ou d'une " grammaire » politique, communes. L'appel aux principes de la Révolution d'octobre par les Tatars ou l'inscription de la mémoire des opposants iraniens dans l' " imaginaire martyropathe » de la République islamique peuvent à la fois, et sans que cela s'oppose nécessairement, indiquer la prégnance d'un univers cognitif commun et l'ambition davantage tactique de prendre l'adversaire au piège de ses propres principes ou valeurs affichés.

Isomorphisme encore dans la manière dont les victimes entreprennent d'élaborer et de promouvoir une histoire alternative à celle des oppresseurs et des vainqueurs. L'enjeu est ici encore de doter une mémoire individuelle, et fortement personnalisée, d'un statut supérieur en généralité, de transformer le récit familial transmis de manière orale en une mémoire collective et officielle à laquelle l'ensemble du groupe puisse s'identifier positivement. Elle aussi marqueur de généralité, la science, en l'occurrence historique, est fréquemment convoquée mais selon des formes qui laissent bien souvent échapper des indices d'une position défavorable dans les rapports de forces tant symboliques que politiques. D'où ces tentatives d'imitation des formes de la production historique institutionnelle, comme dans le recours aux notes de bas de page, bibliographies, titres académiques qui émaillent les récits des épurés - et qui, comme le souligne Caroline Baudinière, annoncent les écrits révisionnistes. D’où également ces recherches obsédées par la question des origines, visant par exemple (cas des Tatars ou des piedsnoirs) à établir l'antériorité de la présence du groupe sur un territoire qui lui est contesté, et par là-même sa légitimité à l'occuper. Se constitue parfois ainsi - Éric Savarese en fournit ici une bonne illustration - ce que l'on pourrait appeler une histoire en simili ${ }^{17}$, dotée de ses maisons d'éditions, canaux de diffusion (librairies, revues, aujourd'hui sites Internet...), figures de proue intellectuelles propres, qui, destinée quasi-exclusivement au public des membres du groupe, se construit et se légitime en large part par imitation de l'univers historique dominant dont elle est précisément exclue.

17. On emprunte cette idée du simili à l'étude des écrivains amateurs, dont les maisons d'édition (à compte d'auteur), concours et associations constituent un « champ littéraire en simili », conduite par Claude F. Poliak, Aux frontières du champ littéraire. Sociologie des écrivains amateurs, Paris, Economica, 2006. 
C'est précisément de cette exclusion que témoigne la recherche de principes de légitimation alternatifs à ceux sur lesquels s'appuie l'expertise de l'histoire dominante et qui, malgré leurs tentatives d'imitation, donne une coloration singulière aux récits historiques victimaires. Le témoignage, ici, s'impose comme une ressource centrale car posée en garante d'une véridicité supérieure à celle de l'expertise historique, suspecte d'incomplétude ou de distorsion. Pour reprendre les catégories d'Elias, au rapport distancié de l'historien aux faits est opposée la connaissance engagée dans les événements du témoin, promu meilleur connaisseur des faits puisque lui les a directement vécus. La connaissance du témoin, pourrait-on dire, est une connaissance par corps, et ce sont les attestations corporelles de cette connaissance qu'il peut se trouver invité à produire comme preuves historiques - comme dans les cas des vétérans Mau Mau dont les cicatrices attestent des souffrances passées dans le même temps qu'elles accroissent leur prestige aux yeux de leurs soutiens. Pour autant, cette connaissance, et la légitimité dont elle se prévaut, n'est pas sans faiblesse, précisément en ce qu'elle s'expose à être renvoyée du côté du singulier et de l'irrationnel. Singularité de l'expérience vécue, tout d'abord, puisque le témoin ne peut raconter que ce qu'il a vu mais est lui-même dépourvu des instruments de généralisation de cette connaissance, et irrationalité lorsque le traumatisme et la souffrance censés définir la victime sont mobilisés pour la disqualifier.

Ici encore, une sociologie des "entrepreneurs de mémoire » s'avère nécessaire afin de comprendre avec quelles motivations (notamment dans le cas de descendants ou proches des victimes directes) et quelles ressources (type et mode d'acquisition du capital culturel, insertion professionnelle...) ceux-ci s'engagent dans la construction d'un récit historique du malheur. D'autres pistes de recherche resteront elles aussi insuffisamment abordées au terme de ce dossier. Les effets - dans plusieurs des textes présentés ici manifestement ambigus - des insertions multiples (dans l'espace organisationnel militant, dans le domaine académique ou ses marges, ou dans le champ du pouvoir) de ces entrepreneurs méritent eux aussi une grande attention, en ce qu'elles déterminent en large part la nature des coups qu'ils sont susceptibles de jouer, les leviers qu'ils mobilisent ou des opportunités dont ils se saisissent. Mériteraient sans doute également une étude plus fine de leur variété les pratiques de la preuve selon les univers sociaux auxquels elle s'adresse : la preuve du journaliste n'est pas toujours celle de l'historien, ni 
celle du juge ou du citoyen critique... Qu'en est-il, dans le processus de construction d'une histoire alternative, des phénomènes de contrebande, d'interférence et d'imitation entre différents registres de la preuve et de l'histoire ? On se limitera à rappeler ce qui devient aujourd'hui un champ d'investigation, en l'occurrence la question des rapports complexes entre registre judiciaire et historique : utilisation de données historiques à charge dans le processus judiciaire ${ }^{18}$, interférences entre droit et histoire qui aboutissent à ce que le droit du passé soit examiné par l'histoire et que le passé soit requalifié aujourd'hui par l'institution judiciaire. Enfin, une sociologie plus fine des historiens et de leur lien à la cause des victimes mériterait d'être posée afin de comprendre par quels canaux peut, ou pas, se mettre en place une histoire alternative.

\section{Des Histoires alternatives ?}

L’Histoire écrite par les victimes, qui souvent prétend faire concurrence aux récits historiques plus légitimes, gouvernementaux et historiens, est-elle d'ailleurs cette Histoire alternative ? Pas toujours si on en croit les auteurs des articles rassemblés ici : si c'est clairement le cas des versions de l'histoire défendues par les épurés évoqués par Caroline Baudinière et par les pieds-noirs étudiés par Eric Savarese, les autres groupes entretiennent un rapport plus ambivalent avec ces récits mieux installés, sinon leur contenu du moins leur forme. Aurélie Campana et Henry Sorg montrent par exemple comment ces " contre-Histoires " s'inscrivent en partie dans la continuité des récits qu'elles contestent : par leur légalisme, ici, par leur parenté avec la valorisation gouvernementale du martyr, là. Ces éléments de continuité, voire ces compromis, attestent la nature pleinement politique des plaintes des victimes ; celles-ci sont en retour, comme le montre Marie-Émmanuelle Pommerolle, largement investies par des stratégies politiques individuelles et collectives. Ce constat amène à nuancer l'hypothèse d'une dépolitisation (qu'alimenteraient le contournement d'une posture politique perçue comme critique, des instances représentatives, et des alliances

18. Sur les risques de «l'historiographie judiciaire » (la réduction des événements ou processus historiques aux seules actions pouvant être rapportées à la responsabilité d'individus, indépendamment des contextes), cf. Carlo Ginzburg, Le juge et l'historien, traduction collective de l'italien, Lagrasse, Verdier, 1997. 
partisanes, ainsi que l'apparence d'une neutralisation des conflits, la mise en avant d'un registre - moral ou humanitaire - donné pour consensuel et l'individualisation du rapport au politique) systématiquement induite par les processus de "victimisation ». La cause des victimes semble certes être mieux servie par des formes dont le contenu politique est mis en sourdine, mais ces formes " dépolitisées » n’en sont pas moins politiques ; souvent, même, les victimes expriment leur réticence à l'égard d'une logique victimaire dépolitisée, préférant utiliser un autre langage (celui par exemple de la résistance) et des formes d'organisation classiques ${ }^{19}$. Les articles réunis ici, et ailleurs ${ }^{20}$, montrent bien que les «mobilisations de victimes », et plus largement les interactions entre ces victimes et leurs interlocuteurs, ne forment pas un ensemble homogène qui participerait d'un air du temps victimaire : le poids des socialisations préalables, le processus de constitution des groupes, leur rapport au politique varient, aussi bien que les objectifs visés (preuve, reconnaissance, réparation - pour reprendre la trilogie évoquée par Fassin et Rechtman - et dans le même temps surcroît de ressources sociales et politiques).

Montrer que les causes des victimes ne s'émancipent pas autant qu'on le croit souvent des contraintes qui pèsent ordinairement sur l'action politique et sociale, ne revient pas à disqualifier la portée morale des mobilisations, mais à rendre compte de la manière dont ces mobilisations s'inscrivent dans l'ordinaire de la vie politique même si elles ne s'y réduisent pas toujours. L'Histoire des victimes n'est pas toujours une contre-Histoire. On peut donc nuancer les hypothèses qui déduisent de la reconnaissance sociale inédite des victimes la modification profonde des formes du récit historien et des modalités de prise de parole publique. Le mimétisme des formes, le recours aux experts, les alliances avec les hommes politiques, limitent cette portée de subversion et confortent les monopoles respectifs des autorités politiques et des professionnels de la production de l'Histoire plus qu'ils ne les minent. Le dispositif des commissions de vérité évoqué plus haut en est un bon exemple : valorisé comme une forme d'expression publique, et cathartique,

19. Voir l'exemple déjà cité des Mères de la place de mai, ou certaines des mobilisations suscitées par l'explosion de l'usine AZF à Toulouse, analysées par Stéphane Latté dans l'ouvrage dirigé par S. Lefranc et L. Mathieu à paraître sur les Mobilisations de victimes.

20. Ibid., et D. Fassin et R. Rechtman, L'Empire du traumatisme, op. cit., par exemple. 
de la vérité des victimes, celui-ci consacre de fait le rôle des experts (historiens, sociologues, juristes, ou anciens hommes politiques), qui déterminent la place, souvent mineure, laissée à cette parole. Le respect dû aux victimes est devenu, sans doute, une évidence sociale, mais il n’a pas libéré les victimes mobilisées du poids des contraintes politiques et sociales ordinaires. 